\title{
Resonance effects in multiple light scattering from statistically rough metallic surfaces
}

\author{
J. A. Sánchez-Gil and M. Nieto-Vesperinas \\ Instituto de Optica, Consejo Superior de Investigaciones Científicas, Serrano 121, Madrid 28006, Spain
}

(Received 8 July 1991; revised manuscript received 4 November 1991)

\begin{abstract}
Multiple scattering of light from one-dimensional random rough metallic surfaces is numerically studied by means of a Monte Carlo simulation method based on the extinction-theorem boundary conditions. Angular and integrated distributions of the mean scattered intensity are computed for several values of the angle of incidence, state of polarization, surface statistical parameters, and dielectric permittivity. Two main regimes are addressed, depending on whether the surface correlation length $T$ is larger or smaller than the wavelength $\lambda$. In the first case, we observe enhanced backscattering both for $s$ and $p$ waves, whereas in the latter situation there exist substantial absorption effects under $p$ polarization, linked to the excitation of surface polaritons. In addition, calculations are made of field enhancements on the surface, owing either to surface-polariton excitation in the small-correlation-length case $(T<\lambda)$, or to multiple scattering and generation of other kinds of surface wave in the large-correlation-length case $(T>\lambda)$.
\end{abstract}

\section{INTRODUCTION}

In this paper we carry out a study of the scattering of light from statistically rough metallic surfaces. We shall pay special attention to the mean scattered (i.e., reflected) intensity, as well as to effects associated with the excitation of surface electromagnetic waves, and, in particular, with surface polaritons (SP).

This classical subject ${ }^{1,2}$ has undergone a considerable advance in recent years. On the one hand, this has been due to the development of controlled experiments from well-characterized samples. ${ }^{3-5}$ On the other hand, Monte Carlo methods have been established for numerically solving the scattering equations for one-dimensional surfaces $z=D(x)$ [namely, $D(x, y)$ being constant in the $y$ direction; the restriction to one-dimensional profiles has been so far imposed by the limitations in computation with a large number of sampling points]..$^{6-19}$ Theory and experiment have led to such results as the phenomenon of enhanced backscattering (also related with that of weak localization of photons in random media ${ }^{20-26}$ transmission effects in thin films and dielectrics, and enhanced specular reflection from symmetric profiles, which were not predictable with previous analytical theories [e.g., the Kirschhoff approximation KA or the perturbation method]..$^{27-38}$

In this work we shall make use of the aforementioned numerical procedures to address multiple light scattering from deeply rough random metallic surfaces with onedimensional profile. The problem is stated in Sec. II. In Sec. III we address the results for the mean scattered intensity. Section IV is devoted to the study of the field on the surface for specific sample profiles. Finally, the main conclusions are briefly synthesized in Sec. V.

Three regimes are considered depending on whether the correlation length $T$ of the surface is larger, equal, or smaller than the wavelength $\lambda$. In the first two cases, we make a comparison, not yet done, with experimental results, ${ }^{5}$ whereas in the latter we discuss effects associated with the excitation of SP under $p$ polarization. ${ }^{39,40}$ These modes have been shown ${ }^{41-43}$ to play a basic role both in the existence of a tiny backscatter peak when $T<\lambda$ and $\sigma<<\lambda$, and also in the existence of substantial absorption effects in the reflected light from gratings. ${ }^{39,40,44,45}$ However, we shall show that the absorption in the case of statistical surfaces is not so strong as observed in metallic gratings, nor it is so critical with the angle of incidence. This is due to the fact that, since a random profile can be considered as a superposition of gratings, many different surface modes are simultaneously present.

Moreover, in studies of light diffraction from metallic gratings, the existence of SP has been linked to that of field enhancements in the selvedge for $p$ polarization. ${ }^{46-49}$ The large values taken on by the field amplitude on metallic surfaces play a fundamental role in, e.g., second-harmonic generation and surface-enhanced $\mathrm{Ra}$ man spectroscopy. ${ }^{46}$ We shall see here that these enhancements also exist in statistical surfaces, although, once again, they are not so dramatic as in the case of gratings. This is discussed in Sec. IV. In this section we shall also analyze the relative influence on these enhancements associated with SP excitation due to both the surface roughness and to the (much weaker) coupling with evanescent waves created by diffraction from the sample edges (also present in flat surface samples).

Finally, we have also observed surface field enhancement for both $s$ and $p$ polarization in surface samples with $T>\lambda$ and large roughness, $\sigma>\lambda$; this last result is as well obtained by means of an iterative treatment up to double scattering of the integral equation governing the scattering from a perfect conductor, as used in the calculation of double, and even triple, scattering to the far field in Refs. 10 and 50-55. On the other hand, this enhancement bears a connection both with previous observations of strong line shapes in the nonradiative region in this range of statistical parameters and polarization, ${ }^{10}$ and with the presence of field enhancements in the selvedge of deep metallic gratings under $s$ polarization, ${ }^{56}$ this sug- 
gesting the existence of some sort of surface electromagnetic waves in this regime.

\section{FORMULATION OF THE SCATTERING PROBLEM}

\section{A. Scattering equations}

The physical problem under consideration is the following: a one-dimensional rough surface $z=D(x)$ separates the vacuum $[z>D(x)]$ from an isotropic and homogeneous medium of dielectric constant $\epsilon(\omega)=\epsilon_{R}(\omega)+i \epsilon_{I}(\omega)$ occupying the half-space $[z<D(x)]$, where $\epsilon_{R}$ and $\epsilon_{I}$ denote real and imaginary parts, respectively.

Upon this surface, a monochromatic, linearly polarized, plane electromagnetic wave impinges at an angle of incidence $\theta_{0}$ with respect to the $z$ axis. Notice that, inasmuch as the surface height does not depend on the $y$ coordinate and the incident wave is linearly polarized with the wave vector in the $x z$ plane, we reduce our initial geometry to a two-dimensional one. Furthermore, no depolarization exists, i.e., $s-\mathrm{TE}$ (respectively, $p$-TM) incident waves cannot be scattered to $p$ (respectively, $s$ ) waves. Consequently, the electromagnetic field is completely described by its transversal component using a scalar treatment. For the sake of simplicity, we define a wave-field function $U(\mathbf{r})$ which stands for the $y$ Cartesian component of the electric (or magnetic) field for $s$ (or $p$ ) polarization. Therefore we write the incident wave field in the form

$$
\mathbf{U}^{(i)}(\mathbf{r})=\hat{\jmath} U^{(i)} \exp \left(i \mathbf{K}_{0} \cdot \mathbf{r}\right)
$$

In Eq. (1) $\mathbf{r}=(x, z), \hat{\mathbf{j}}$ is the unit vector along $O Y$. We denote the incident and reflected wave vectors as $\mathbf{K}_{0}$ and $\mathbf{K}$, respectively,

$$
\begin{aligned}
& \mathbf{K}_{0} \equiv\left(K_{0},-q_{0}\right)=k_{0}\left(\sin \theta_{0}, 0,-\cos \theta_{0}\right) \\
& \mathbf{K} \equiv(K, q)=k_{0}(\sin \theta, 0, \cos \theta)
\end{aligned}
$$

where

$$
|\mathbf{K}|^{2}=\left|\mathbf{K}_{0}\right|^{2}=k_{0}^{2}=\frac{\omega^{2}}{c^{2}}=\left[\frac{2 \pi}{\lambda}\right]^{2},
$$

$\lambda$ being the wavelength of the incident plane wave. $\theta_{0}$ and $\theta$ are the incidence and observation angles above the surface. As customary, we omit a time-dependent factor $\exp (-i \omega t)$ in the fields throughout.

We aim to determine the field scattered from the rough metallic interface $\left[\epsilon_{R}(\omega)<0, \epsilon_{I}(\omega)>0\right]$. By using the extinction theorem ${ }^{35-37}$ as a nonlocal boundary condition, we obtain the scattered field in the vacuum region in terms of the source functions $U(x)$ and $W(x)$, which are defined by

$$
U(x)=\left\{\begin{array}{l}
U^{\text {(out) }}(x, D(x)) \\
U^{(\text {in })}(x, D(x)),
\end{array}\right.
$$

$$
W(X)=\left\{\begin{array}{l}
\gamma\left(\frac{\partial U^{(\text {out })}(\mathbf{r})}{\partial n}\right]_{z=D^{(+)}(x)} \\
\frac{\gamma}{\alpha}\left[\frac{\partial U^{(\mathrm{in})}(\mathbf{r})}{\partial n}\right]_{z=D^{(-)}(x)}
\end{array}\right.
$$

where the continuity relations across the interface have been accounted for. The superscripts (out) and (in) mean inside vacuum and metal, respectively. $D^{(+)}$and $D^{(-)}$ denote the surface profile when approached from above (vacuum) and below (metal), respectively. The normal derivative is $\partial / \partial n \equiv \hat{n} \cdot \nabla, \hat{n}$ being the local outward normal vector

$$
\hat{n} \equiv \frac{1}{\gamma}(\{-d[D(x)] / d x\}, 1),
$$

where $\gamma=\left(1+\{d[D(x)] / d x\}^{2}\right)^{1 / 2}$. The constant $\alpha$ depends on the polarization

$$
\begin{aligned}
& \alpha=1 \text { for } s \text { polarization , } \\
& \alpha=\epsilon(\omega) \text { for } p \text { polarization . }
\end{aligned}
$$

The source functions $U(x)$ and $W(x)$, as given by Eqs. (4) and (5), as well as the far field and its corresponding intensity, are obtained following the analysis and the numerical method described in Refs. 10 and 16.

\section{B. Surface model}

We assume for the surface-profile function $z=D(x)$ a statistically homogeneous and isotropic random process with zero mean $[\langle D(x)\rangle=0]$ and normal probability density function, $\sigma$ being its rms deviation. In addition, a Gaussian correlation function $c(\tau)$ is considered as follows:

$$
c(\tau)=\frac{1}{\sigma^{2}}\langle D(x) D(x+\tau)\rangle=\exp \left(-\frac{\tau^{2}}{T^{2}}\right) .
$$

$T$ denotes the correlation length.

We apply the Monte Carlo method employed in Ref. 19 to simulate the surface profile (see also Refs. 8 and 16). In this procedure, a sequence of random numbers, uniformly distributed between $[0,1]$, and directly generated by the computer, is transformed into a sequence (typically $10^{5}$ numbers) with normal statistics, zero mean, and unity variance. Finally, in order to obtain a correlation function as given by Eq. (8), the latter sequence is correlated with a Gaussian function with the appropriate width $T$, and scaled to the desired $\operatorname{rms} \sigma$.

\section{Numerical calculation}

Considering a surface sample of length $L$ consisting of $N$ points, and a plane wave, either $s$ or $p$ polarized, incident at a given angle $\theta_{0}$ with the normal to the mean plane, then, by means of a quadrature scheme, the integration over the surface is converted into a summation from $n=1$ to $N\left[x_{n}=-L / 2+(n-1) L / N\right] .{ }^{10,16}$

After repeating the same process for a certain number 
of samples, and averaging the squared modulus of the resulting far fields, the mean scattered intensity is calculated for a given incident angle $\theta_{0}$. It is useful to carry on the calculation for both $\theta_{0}$ and $-\theta_{0}$. In this way, we manage to double the effective number of samples over which the average is performed, without doubling the computer time. ${ }^{8}$ It should be mentioned that, as expected, this procedure does not alter significantly the angular distribution of mean intensity with respect to that obtained at $\theta_{0}$ only, but contributes indeed to a faster convergence of the results when the number of samples is increased. Moreover, it constitutes a simple way of symmetrizing the scattered far field for normal incidence.

Calculations were carried out on a CDC-Cyber $180 / 855$ computer and on a VAX 9210 computer. We employ from 220 up to 300 sampling points for each profile (we have checked that, generally, the choice of 300 points does not appreciably modify the results). Besides, three different regimes of surface correlation length $T$ are submitted to study as both the angle of incidence $\theta_{0}$ and the rms $\sigma$ vary. For $T \geq \lambda$, the surface profiles have a length $L=72.81 \lambda$. In this case, our choice of $N=300$ yields about ten sampling points per wavelength, which is adequate for accuracy of the numerical results. ${ }^{8,9}$ For $T<\lambda, L=22 \lambda$, and using $N=220$ implies ten sampling points per wavelength, but two points per correlation length. Even so, the results exhibit a high enough degree of accuracy and consistency, as checked on comparison with other authors' computations, ${ }^{4,5,9,10}$ among whom some take a much larger sampling point record (cf. Ref. 58) by means of a very wide beam simulation method. The minimum effective number of surface records over which the average is performed is 200 . We have observed, however, that making this number double or triple indeed smooths the angular distributions of scattered intensity, though the main features are not significantly modified. Generally, the following angles of incidence are considered: $\theta_{0}=0^{\circ}, 10^{\circ}, 20^{\circ}, 30^{\circ}, 40^{\circ}, 50^{\circ}, 60^{\circ}$, and $70^{\circ}$. The adequacy of the results, inherently noisy due to the limited number of samples in the averaging, is tested on the basis of different criteria. First, the numerical convergency of the mean intensity as the number of surface samples increases. Second, the mean total reflectance $R$ can by no means exceed the unity value. This constitutes the energy conservation criterion. Third, the results calculated with different lengths $L$ should be consistent with each other. Finally, we check the reciprocity law: The scattered intensity for the fixed direction of incidence $\mathbf{K}_{0}$ and of observation $\mathbf{K}$ must be equal to that for the incident direction $-\mathbf{K}$ and of observation $-\mathbf{K}_{0}$. This law is readily derived from the invariance of the scattering matrix under time reversal.

As shown in Ref. 8, the choice of a Gaussian beam ${ }^{10}$ instead of a plane wave as incident field does not significantly affect the results; the noise due to the averaging process is generally larger than that introduced by edge effects. Only in the case where $T$ and $\sigma$ are smaller than $\lambda$, and there exists an intense specular peak, the side lobes of this peak can swamp the values of the diffuse halo in the vicinity of the specular direction; nevertheless, this is avoided by subtracting the coherent part in order to uniquely evaluate the angular distribution of the diffuse component.

\section{RESULTS FOR THE MEAN SCATTERED INTENSITY}

In this section we report the numerical results of our scattering simulation for the angular distribution of mean scattered intensity and of its diffuse component as well as for the reflectance. ${ }^{16}$ We have calculated these values for three different dielectric constants $\epsilon(\omega)$ in the metallic region: ${ }^{59} \epsilon(\omega)=-4.12+i 1.29$ (gold at $\lambda=516.6 \mathrm{~nm}$ ), $\epsilon(\omega)=-9.89+i 1.05$ (gold at $\lambda=652.6 \mathrm{~nm}$ ), and $\epsilon(\omega)=-17.2+i 0.498$ (silver at $\lambda=652.6 \mathrm{~nm}$ ).

\section{A. Large correlation length $(T>\lambda)$}

Within this regime, we chose $T=3.16 \lambda$ in order to compare our numerical results with the existing measurements reported in Ref. 4 and 5. Figure 1 shows the angular distribution of mean scattered intensity from a gold surface with $\epsilon=-9.89+i 1.05, T=3.16 \lambda$, and $\sigma=1.9 \lambda$. In what follows, we denote the backscattering direction by a solid vertical line on the left, whereas a dashed vertical line on the right is used to indicate the specular direction. Note that, owing to the large roughness, no coherent component appears. By contrast, a remarkable peak arises in the backscattering direction, which is clearly observable for $\theta_{0}$ up to $30^{\circ}$ in $p$ polarization, and for $\theta_{0}$ up to $40^{\circ}$ in $s$ polarization. For the sake of brevity, we only plot here the curves for $40^{\circ}$.

The results of Fig. 1 agree well with the experiments performed with one-dimensional gold surfaces with the same statistical parameters as here (cf. Figs. 8 and 9 in Ref. 5, taking into account the different convention there for incidence and observation angle and for the normalization). It should be pointed out that if the medium is as-

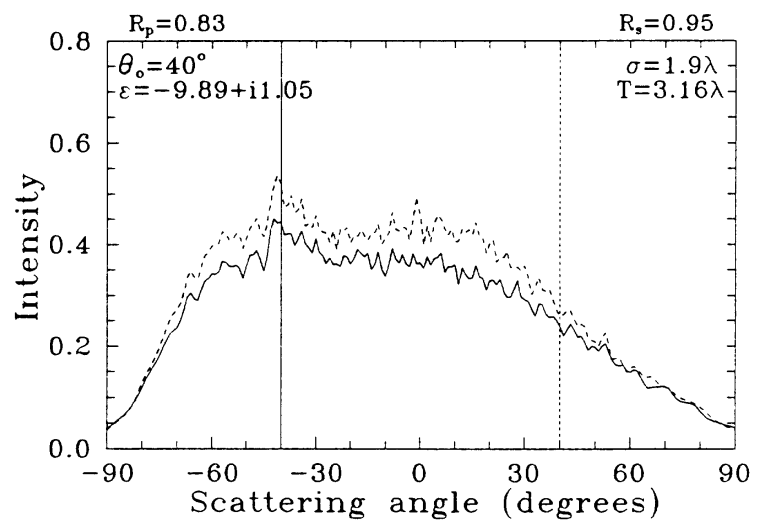

FIG. 1. Angular distribution of mean scattered intensity $\langle I(\theta)\rangle / I_{0}$ from a metal surface with $T=3.16 \lambda, \sigma=1.9 \lambda$ and $\epsilon=-9.89+i 1.05$, at $\theta_{0}=40^{\circ}$, averaged over 400 samples. Dashed line: $s$ polarization. Solid line: $p$ polarization. The average reflectance for each polarization is a shown. The specular direction is marked by the dashed vertical line. The backscattering direction is shown by the solid vertical line. 
sumed to be a perfect conductor, the resulting numerical calculation using the extinction theorems (ET) exhibits a considerable departure from the experimental curves for increasing angle of incidence. In fact, the angular distributions for perfect conductors at such angles of incidence behave as quasi-Lambertian for both polarizations. ${ }^{5,8}$ By contrast, those for a real metal resemble more accurately the tendency towards the backscattering direction manifested by the experimental curves, and reproduce the slight differences existing between $s$ and $p$ polarization. A comparison between our numerical calculations (Fig. 1) and the aforementioned experimental results at $40^{\circ}$ yields a degree of agreement surpassing those of numerical results similar to that of Fig. 1 which have been previously reported $^{5,10,58}$ regarding this regime of surface statistical parameters.

If we change the dielectric constant of the metal, the behavior of the angular distributions of scattered intensity for $p$ waves is slightly more affected than for $s$ waves; however, the backscatter peak structure is preserved in spite of the larger or smaller height reached by the peak, which depends on the degree of absorption undergone by the incident beam. This narrow peak has been found even in dielectric interfaces with high enough permittivity $(\epsilon \geq 4) .4,10,16,17$

Figure 2 shows the average reflectance versus rms $\sigma$ for the three dielectric constants mentioned above, keeping $T=3.16 \lambda$ and $\theta_{0}=20^{\circ}$ fixed. The reflectance exhibits a very slight and monotonous decrease as $\sigma$ increases. Moreover, although not shown here, the reflectance versus $\theta_{0}$ for a particular choice of $T, \sigma$ and $\epsilon$ follows the plane $(\sigma=0)$ result, given by the Fresnel coefficients for $\epsilon(\omega)$ (cf. Ref. 60), but slightly shifted downwards by an amount that increases with $\sigma$. This behavior looks reasonable on the basis of geometrical optics (GO). The net absorption characterized by the imaginary part of $\epsilon(\omega)$ is related to the number of hits suffered by the incident wave. Double scattering introduces an event of absorption onto the metallic surface additional to that of single scattering. Therefore the absorption slightly increases with $\sigma$, thus decreasing the reflectance. Besides,

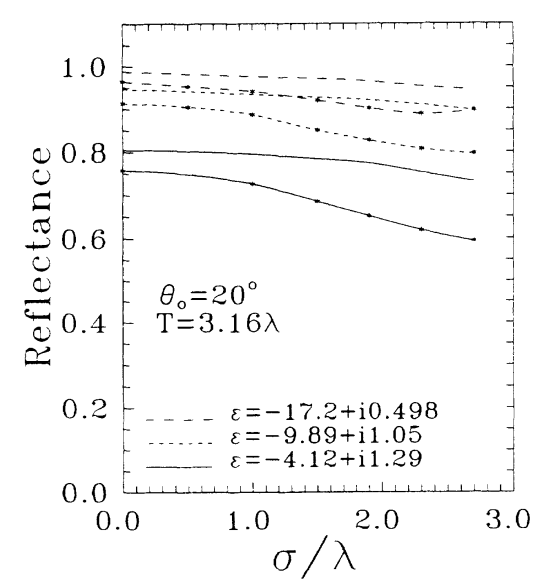

FIG. 2. Average reflectance from 200 samples vs roughness $\sigma / \lambda$ for $T=3.16 \lambda$ at $\theta_{0}=20^{\circ}$. With asterisks $p$ polarization; unadorned, $s$ polarization. we have observed that changing the angle of incidence does not modify significantly the absorbed energy by a given rough surface, since the number of scattering events hardly varies with $\theta_{0}$ (except near grazing incidence, at which there exists only specular reflection).

\section{B. Correlation length equal to the wavelength $(T=\lambda)$}

Qualitatively, the angular distributions of mean scattered intensity for $T=\lambda$ do not differ too much from those for $T=3.16 \lambda$, previously studied before. In particular, enhanced backscattering occurs provided that $\sigma$ takes on a large enough value. For instance, $\sigma=0.5 \lambda$ ensures the existence of a backscatter peak for $T=\lambda$. Logically, the peak is wider than that for $T=3.16 \lambda$, since its angular width depends on $\lambda / T .^{3,10}$

Figure 3 shows the reflectance versus $\sigma$ for $T=\lambda$ and $\theta_{0}=20^{\circ}$. If one excludes the $p$ polarization result for the most absorbing metals $(\epsilon=-4.12+i 1.29$ and $\epsilon=-9.89+i 1.05)$, the curves resemble those of Fig. 2. Roughly speaking, the explanation of the absorption given in connection with Fig. 2 also holds within this regime of $T$. Nevertheless, a stranger contribution to the absorption of energy arises in the case of Fig. 3, because of the dissipation due to the excitation of SP. ${ }^{39,40}$ These elementary surface modes can be only excited by $p$ waves. Once a SP is excited, it propagates along the surface until it either becomes attenuated [owing to intrinsic loss mechanisms within the metal such as interband transitions, which are enclosed in $\left.\epsilon_{I}(\omega)\right]$ or decays into a radiative mode (photon). For an infinite flat surface, an incident photon cannot couple to a SP because their dispersion relations never cross each other ${ }^{39,40}$ The probability of excitation becomes nonzero if roughness is introduced on the surface. Moreover, within an interpretation based on a perturbative theory for weakly rough surfaces, this probability is proportional to the power spectral density of the surface ${ }^{33,34,61-63}$ (namely, to $\exp \left(-K_{\mathrm{SP}}^{2} T^{2}\right)$ for a Gaussian correlation function as given by $\mathrm{Eq}$. (8), where $\mathbf{K}_{\text {SP }}$ is the SP wave vector). Although this formulation is only valid for $T<\lambda$ and $\sigma \ll T$, it still provides a qualita-

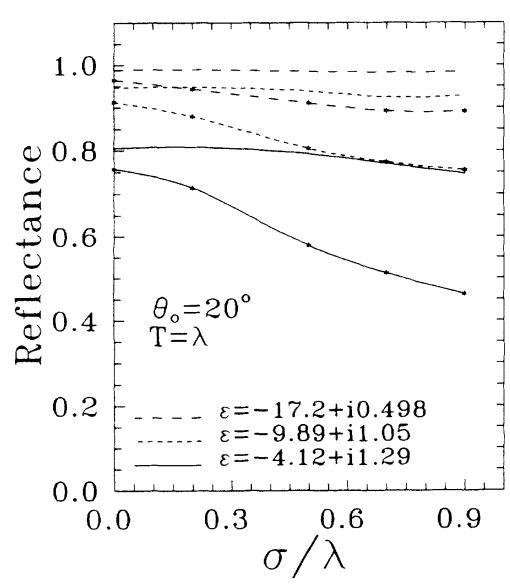

FIG. 3. Same as Fig. 2 for $T=\lambda$. 
tive idea of the dependence on $T$ of the SP probability of excitation. The case $T=3.16 \lambda$, seen in Sec. III A, gives an almost negligible probability of excitation, ${ }^{41}$ as implied from a comparison between the reflectances for $p$ and $s$ polarization in Fig. 2. Figure 3 reveals that $p$-wave absorption supers $s$-wave absorption. We interpret this additional amount of absorbed energy for $p$ polarization as due to the roughness-induced excitation, and subsequent damping, of SP, which is not possible for $s$ polarization. However, as this extra absorption is relatively small, we can infer that the coupling photon SP is weak in this regime of $T$. By contrast, the excitation of SP does play a more relevant role in the scattering from surfaces with $T \ll \lambda$, as we will see below.

\section{Subwavelength correlation length $(T<\lambda)$}

The surface-correlation length considered throughout this section takes on the value $T=0.2 \lambda$. The rms $\sigma$ ranges from $\sigma=0$ to $0.3 \lambda$. For $\sigma \geq 1.5 T$ our numerical method starts to fail.

In Refs. 41-43 enhanced backscattering for $p$ polarization was predicted for a surface with $\sigma=0.01 \lambda$ through a diagrammatic addition of multiple-scattering contributions using both the Rayleigh hypothesis ${ }^{1,2}$ and the ET expressions. ${ }^{35}$ Later on, calculations based on the numerical solution from the ET confirmed the former prediction. ${ }^{9,10}$ The physical mechanism involved in producing the backscatter peak was interpreted on the basis on the excitation and deexcitation of SP. ${ }^{41,42}$ Since an $s$ polarized photon cannot couple to a SP (this only holds in one-dimensional surfaces), no backscatter peak appears for $s$ polarization.

In this work we concentrate on the area enclosed by the angular distribution, namely, the reflectance for $s$ and $p$ waves. In Fig. 4 the total (diffuse plus specular) reflectance versus the angle of incidence is plotted for $\epsilon(\omega)=-9.89+i 1.05(T=0.2 \lambda, \sigma=0.1 \lambda)$, including the reflectance for a plane (Fresnel coefficient). The net

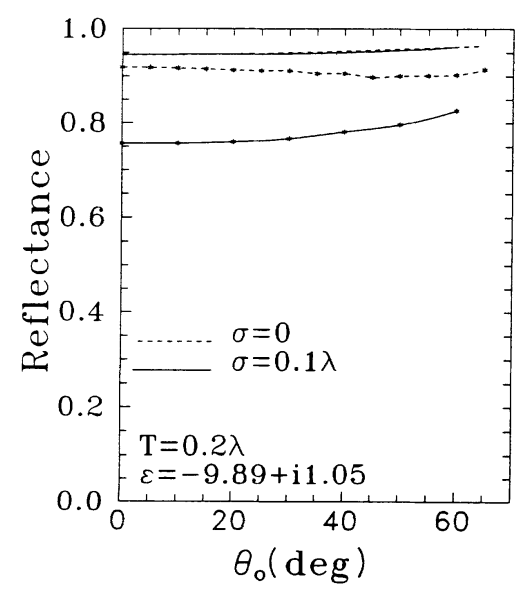

FIG. 4. Average reluctance from 200 samples vs $\theta_{0}$ for $T=0.2 \lambda, \sigma=0.1 \lambda$, and $\epsilon=-9.89+i 1.05$ (solid lines). The result for a plane ( $\sigma=0$, dashed lines) is also included. With asterisks, $p$ polarization; unadorned $s$ polarization. roughness-induced absorption for $p$ polarization, obtained on substracting the rough surface result from the flat surface result, is maximum at normal incidence and decreases continuously as $\theta_{0}$ increases. For $s$ polarization, it is very similar for both metals, and does not depend on $\theta_{0}$. The behavior of the reflectance for $p$ waves agrees fairly well with that expected when the metal surface supports SP. ${ }^{33,34}$

Figure 5 shows the total reflectances versus $\sigma$ for the three metals, with $T=0.2 \lambda$ at $\theta_{0}=20^{\circ}$. Whilst the $s$ polarization result is not modified by the roughness, the $p$ polarization reflectance presents an outstanding structure. Initially, it decreases with the roughness. At a certain value of $\sigma$, which depends on $\epsilon$, a minimum is reached. Then the reflectance begins to grow again as $\sigma$ increases further. A physical explanation of this behavior can be given in terms of the excitation of SP. ${ }^{49}$ When a slight roughness is ruled on a metal surface, light weakly excites SP, which are subsequently damped transferring their energy within the metal on propagating along the surface. As the roughness increases, so does the probability of excitation, thus increasing the absorption through these surface modes. However, the larger $\sigma$ is, the more probable it becomes that SP are scattered by coupling with propagating modes and thus that photons are thrown back to the vacuum, producing an increase in the reflectance. The minimum (respectively, the maximum) value of the reflectance (respectively, of the absorption curve) is due to a balance between both processes: excitation (and then dissipative damping) and reradiation of SP. If $\sigma$ exceeds this value at which there is maximum absorption, radiative damping prevents SP from being efficiently excited, and subsequently dissipated: thus the reflectance gradually increases. Finally, beyond a certain high value of the roughness $\sigma$, the radiative daming should be so strong that the coupling between photons and SP would become virtual; namely, SP would be simultaneously created and destroyed so that, in practice, no excitation would exist. Our calculation method does not permit us to achieve accurate results for such rough surfaces $(\sigma / T \geq 2)$, but we infer from our results that the reflectance would then tend to a value similar to the one

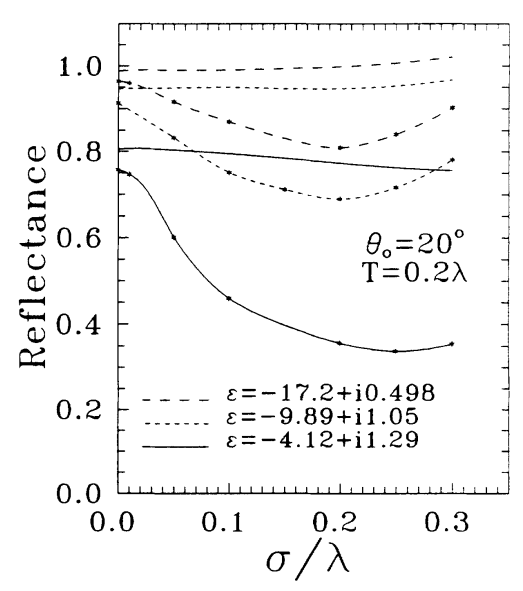

FIG. 5. Same as Fig. 2 for $T=0.2 \lambda$. 


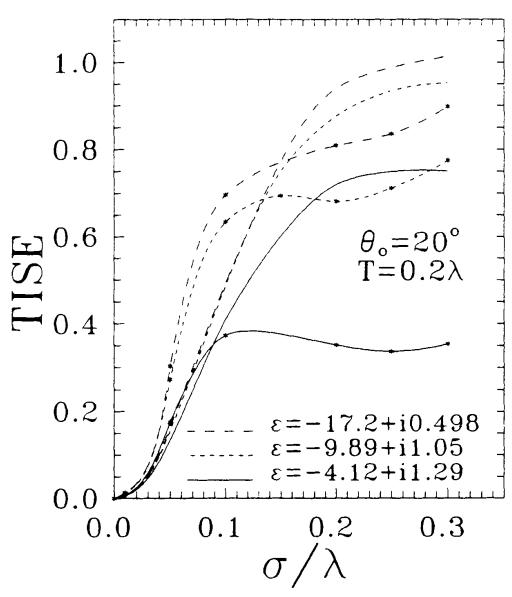

FIG. 6. Same as Fig. 5 for the mean total incoherently scattered energy (TISE) in arbitrary units.

it takes on for $\sigma=0$.

If we wish to analyze the incoherent part, then the mean total incoherently scattered energy (TISE) must be calculated. Figure 6 shows the TISE versus $\sigma$ for the three metal dielectric constants already used, keeping $T=0.2 \lambda$ and $\theta_{0}=20^{\circ}$ fixed. The curves for $s$ polarization manifest the expected behavior: the TISE, which is zero for a plane surface, grows as the roughness increases, unlike the coherent part, which simultaneously decreases with $\sigma$. Beyond $\sigma=0.2 \lambda$ the scattered energy consists only of the incoherent component: This is evident by comparing the TISE with the reflectance (Fig. 5). For $p$ polarization the absorption due to the excitation of SP affects considerably the behavior of the TISE. About the regime of roughness where maximum absorption takes place, a relative minimum of the TISE is observed. Nevertheless, if the coherent component is calculated by substracting the TISE in Fig. 6 from the reflectance in Fig. 5 , we find a very similar dependence on $\sigma$ for both polarizations. This indicates that the effects of the absorption of light through surface modes take place mainly for the diffusely scattered waves.

\section{SURFACE-FIELD ENHANCEMENTS}

So far, we have discussed the distribution of scattered field above the selvedge. In this section the values of the wave field on the surface, given by the functions $U(x)$ and $W(x)$, are submitted to study. The underlying physical mechanisms that give rise to phenomena such as enhanced backscattering or excitation of SP may be interpreted in connection with the total field on the surface, resulting from the scattering process. In fact, in the case of light diffraction from gratings, it is well known that the absorption of light due to the excitation of SP is linked to the existence of field enhancements in the selvedge and, in particular, in the surface. ${ }^{39,46,48,49}$

In the case of a perfect conductor, the continuity conditions impose that the tangential component of the electric field and the normal component of the magnetic field must both vanish on the surface. Therefore, as a real metal approaches a perfect conductor, those quantities tend to zero. We shall then define a surface current function $J(x)$ as, either the normal derivative of the total electric field for $s$ polarization, or the total magnetic field for $p$ polarization, both evaluated on the surface. Namely,

$$
\begin{aligned}
& J_{s}(x)=\frac{1}{k_{0}} W(x), \\
& J_{p}(x)=U(x) .
\end{aligned}
$$

The function $J(x)$ allows us to compare quantitatively surface fields of real metals and of perfect conductors, when necessary. Throughout this section, we shall address $J(x)$ as obtained from the system of coupled integral equations of Refs. 10 and 16 for different segments (of length $L$ ) of surface profiles.

\section{A. Flat surface}

We shall make first a theoretical analysis to give some insight into the physical mechanisms underlying the numerical results; then we shall discuss the consistency of this interpretation with the exact numerical computations. For a infinite planar interface, the reflected surface field is the incident field on the surface multiplied by the corresponding Fresnel coefficient. If the metallic illuminated interface $z=0$ has a finite length $L$, then the reflected surface field will be altered near the edges by diffraction (let us denote the edge points by $x=a$ and $x=-a$, with $L=2 a$ ). By means of the angular spectrum representation, the scattered field for $z>D_{\max }\left[D_{\max }\right.$ being the maximum value of $z=D(x)]$ may be written in the form

$$
U^{(r)}(\mathbf{r})=\int_{-\infty}^{\infty} A(K) e^{i(K x+q z)} d K
$$

For a finitely extended incident plane wave, the angular spectrum of the field reflected at the flat surface will be

$$
A(K)=\mathscr{R}(K) F\left(K, K_{0}\right),
$$

where $F\left(K, K_{0}\right)$ has the following shape:

$$
F\left(K, K_{0}\right)=\frac{1}{\pi} \frac{\sin \left[\left(K-K_{0}\right) a\right]}{K-K_{0}} .
$$

$a$ is half the length of the surface, namely $a=L / 2$. In Eq. (11) $R(K)$ denotes the Fresnel reflection coefficient (cf. Ref. 60), which depends on the polarization as follows:

$$
\begin{aligned}
& \mathcal{R}_{s}(K)=\frac{q-q^{\prime}}{q+q^{\prime}} \text { for } s \text { polarization, } \\
& \mathcal{R}_{p}(k)=\frac{\epsilon(\omega) q-q^{\prime}}{\epsilon(\omega) q+q^{\prime}} \text { for } p \text { polarization } .
\end{aligned}
$$

$K$ and $q$ are defined in Eqs. (2) and $q^{\prime}=\left[\epsilon(\omega) k_{0}^{2}-K^{2}\right]^{1 / 2}$. It should be remarked that the equivalence made in this theoretical analysis between illuminating an infinite surface with a truncated plane wave and illuminating a truncated surface is not strictly rigorous. One should introduce currents on the surface edges to correctly account 
for edge-diffraction effects. We have glossed over this so that in fact our theoretical analysis based on Eq. (11) would actually correspond to using the KA for the edgediffraction effect. Since the extent of the illuminated surface is much larger than the wavelength $(L=22 \lambda)$, we expect this approximation not to introduce a too strong quantitative error in our theoretical interpretation. A similar approach has been previously used, e.g. in Eqs. (6.4) and (6.5) of Ref. 10.

For $p$ polarization, the presence of resonant poles in the Fresnel coefficient Eq. (13b) at $K= \pm K_{\text {SP }}$ produces a weak excitation of SP. The integration of the equation, resulting from introducing Eqs. (11) and (12) into Eq. (10), is done in the Appendix, and yields the scattered field. By adding this result to the incident field $U^{(i)}$, introducing it into Eq. (10), and evaluating both at $z=0$, we finally obtain (see Appendix):

$J_{p}(x)=\left\{\begin{array}{rr}S_{--}(x)+S_{+-}(x), & x<-a \\ {\left[1+\mathcal{R}\left(K_{0}\right)\right] U^{(i)}(x)+S_{-+}(x)+S_{+-}(x),} & -a \leq x \leq a \\ S_{-+}(x)+S_{++}(x), & x>a .\end{array}\right.$

$S_{--}(x), S_{+-}(x), S_{-+}(x)$, and $S_{++}(x)$ are surface waves (SP), as defined in the Appendix [cf. Eqs. (A6), (A7), (A10), and (A13) evaluated at $z=0$ ]. They are the result of the coupling between evanescent waves produce by edge diffraction due to the surface-illumination truncation and the resonant-surface modes associated with poles of $\mathscr{R}_{p}(K)$. Four SP are created near the point $x=a$ (respectively, $x=-a$ ) in the boundary, which propagate in the forward direction $S_{++}(x)$ [respectively, $S_{-+}(x)$ ] and in the backward direction $S_{+_{-}}(x)$ [respectively, $\left.S_{--}(x)\right]$. Within the surface, the field contains also the incident and specularly reflected fields [cf. Eq. (14b)].

For $s$ polarization, the absence of poles implies that no SP are excited. The function $J_{s}(x)$ then consists only of incident and specularly reflected components

$$
J_{s}(x)=\left\{\begin{array}{l}
\frac{1}{k_{0}}\left[1-\mathcal{R}\left(K_{0}\right)\right] W^{(i)}(x), \quad|x| \leq a \\
0, \quad|x|>a
\end{array}\right.
$$

where $W^{(i)}(x)$ is the normal derivative of the incident field - a plane wave-evaluated at $z=0$, i.e.,

$$
W^{(i)}(x)=-i q_{0} U^{(i)}(x)=-i q_{0} e^{i K_{0} x} .
$$

Equations (15) have been calculated following the same procedure as in the Appendix for the electric field, but without poles at $K= \pm K_{\mathrm{SP}}$, and then taking the normal derivative.

In order to confirm this analytical interpretation based on the angular spectrum representation of the wave fields, $J(x)$ [Eqs. (9)] is computed by numerically solving the exact (ET) equations. ${ }^{16}$ In Fig. 7 the modulus of $J(x)$ is shown in the zone $|x| \leq a=11 \lambda$ for $\epsilon=-9.89+i 1.05$ at normal incidence. For $s$ waves, the edge effects are

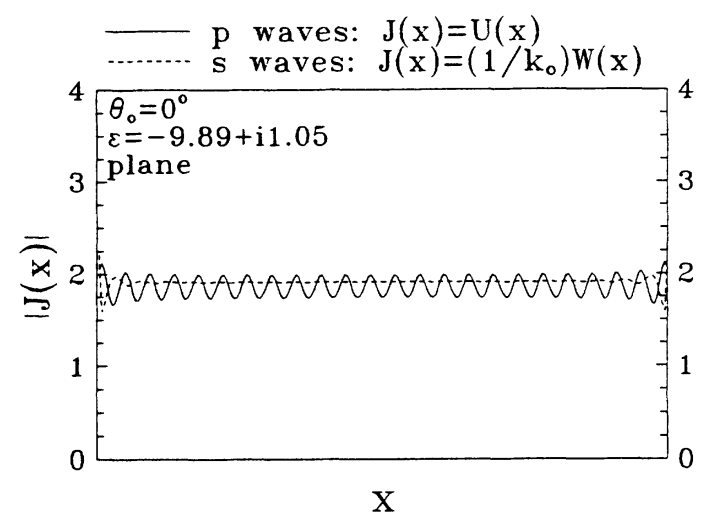

FIG. 7. Modulus of the surface current function $J(x)$ for a planar surface of length $L=2 a=22 \lambda$ for $\epsilon=-9.89+i 1.05$ at normal incident. Dashed line: $s$ polarization. Solid lines: $p$ polarization.

practically negligible. As expected, $J_{s}(x)$ yields a modulus close to 2 [exactly, $1-\mathcal{R}_{s}\left(K_{0}\right)$, cf. Eq. (13a), $\mathcal{R}_{s}\left(K_{0}\right)=-1$, and $\mathcal{R}_{p}\left(K_{0}\right)=1$ for a perfect conductor] and a phase, not depicted here, about $-\pi / 2$ due to the $-i$ factor in Eq. (16). For $p$ waves, the interference between the weakly excited SP $S_{-+}(x)$ and $S_{+_{-}}(x)$ gives rise to a standing wave, clearly seen from the oscillations in $J_{p}(x)$. These oscillations have small amplitudes, and are centered about the values $1+\mathcal{R}_{p}\left(K_{0}\right)$ for the modulus, and about 0 for the phase, as predicted by Eqs. (14). According to Eqs. (A7) and (A10), the wave vectors of these surfaces modes should be, respectively, $-K_{\mathrm{SP}}$ and $K_{\mathrm{SP}}$. This coincides with the observed frequency of the standing wave in Fig. 7 through the real part $\left(K_{\mathrm{SP}}^{(R)}\right)$ of $K_{\mathrm{SP}}$. Although not shown here, we have also analyzed the behavior of $J(x)$ for $\epsilon=-4.12+i 1.29$. In this metal, the SP attenuation length, which is equal to $\left(2 K_{\mathrm{SP}}^{(I)}\right)^{-1}$ ( $K_{\mathrm{SP}}^{(I)}$ is the imaginary part of $K_{\mathrm{SP}}$ ), is very short. The SP exponential decay takes place in a few wavelengths in such a way that $S_{+-}$and $S_{-+}$almost disappear before they cross each other. This dissipative damping becomes really relevant when SP are more efficiently excited through surface roughness $(T<<\lambda)$, thus producing a large light absorption rate.

\section{B. Surface-field enhancements due to the excitation of SP via roughness}

Let us consider a rough surface in the regime $T<\lambda$ where the excitation of SP is significant and thus plays a fundamental role. We simulate a surface record of length $L=2 a=22 \lambda$ by extracting a segment of random numbers from a sequence with $T=0.2 \lambda$ and $\sigma=0.05 \lambda$. Then $J(x)$ is obtained from Eqs. (9a) and (9b), with $U(x)$ and $W(x)$ numerically calculated following Refs. 10 and 16 . Figure 8 shows the modulus and the phase of $J(x)$ for $\epsilon=-9.89+i 1.05$ at normal incidence. The surface profile in an arbitrary scale is included in between the graphs. A comparison with Fig. 7 indicates that the influence of the surface edges on the field enhancements is much smaller than that of the roughness. Also, we ob- 
serve huge differences between both polarizations. The surface current for $s$ waves is very slightly modified by the roughness, keeping an almost constant amplitude of $\left[1-\mathcal{R}_{s}\left(K_{0}\right)\right]$ and a phase with value about $-\pi / 2$, both modulated by the shape of the surface. In contrast, $J_{p}(x)$ presents irregular oscillations of very large amplitude that we interpret as strong SP excited through roughness. In fact, we observe that the frequency of these oscillations is related on average with the corresponding value of $K_{\mathrm{SP}}^{(R)}$ (cf. Ref. 10), and seems to be practically independent of the surface shape. This point is confirmed by the results obtained for the other metallic permittivities mentioned in the preceding section. As seen in Fig. 8, these SP induce remarkable enhancements of the surface field. In terms of $|J(x)|^{2}$, enhancements of the order of 25 (normalized to the incident field) are encountered at certain points of the surface. The enhancement decreases on average as the dissipative damping associated with $\epsilon_{I}$ increases. This is closely linked with the great losses undergone by the total reflectance for the most absorbing metals discussed in Sec. III C (cf. Fig. 5). This phenomenon has been studied in gratings, ${ }^{46-49}$ where large reflectance
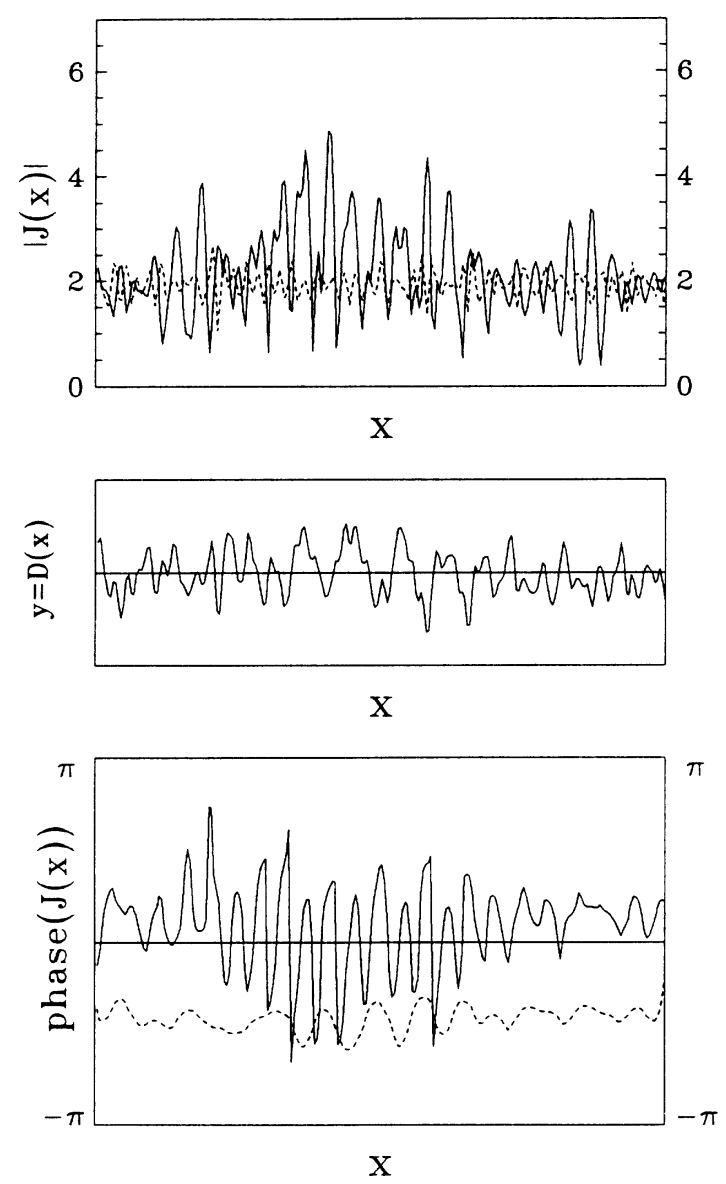

FIG. 8. Modulus and phase of $J(x)$ for a sample of length $L=2 a=22 \lambda$ extracted from a sequence with $T=0.2 \lambda$ and $\sigma=0.05 \lambda ; \theta_{0}=0^{\circ}$. The sample profile in an arbitrary scale is included in between. Dashed line: $s$ polarization. Solid line: $p$ polarization. drops and enhancements of the surface field occur when a nonpropagating diffraction order coincides with a SP. On the other hand, the surface current calculated for $\epsilon=-4.12$, not plotted in Fig. 8, reveals that indeed, when the dissipative losses are minimized, the enhancement for $p$ waves reaches its maximum value; in this case, the total reflectance, which has not been shown in Sec. III C, yields a value similar to that for a flat surface, so that no light absorption appears $\left(\epsilon_{I}=0\right)$.

\section{Surface-field enhancements for $T>\lambda$}

We shall next consider rough surfaces with $T>\lambda$. As in Sec. IV B, a surface record of length $L=2 a$ is chosen from a sequence of random numbers with given $T>\lambda$. The surface current $J(x)$ induced on such a surface when light impinges upon it at different angles of incidence is numerically obtained once again from Eqs. (9) and according to Ref. 16.

In order to analyze the influence of multiple scattering in this regime of statistical parameters, and hence, the appearance of surface-field enhancements in those situations in which there is enhanced backscattering, we shall compare the numerical results obtained from the extinction theorem with those based on an iterative treatment of the scattering equations corresponding to a perfectly conductive surface. ${ }^{10,50-53,55}$

Let us consider a surface sample with $T=3.16 \lambda$, $\sigma=0.5 \lambda$, and $L=2 a=27.81 \lambda$. In this range of values of $T$ and $\sigma$, the KA is valid and, thus, it gives an accurate description of the scattering process. ${ }^{14} \mathrm{~A}$ comparison between the numerical results for the surface current $J(x)$ for real metals and the KA for perfect conductors, not plotted here, manifests a good agreement except for the SP weakly excited near the edges of the metallic surfaces (cf. Fig. 7).

If the roughness is further increased, the double scattering becomes relevant. Figure 9 shows $|J(x)|$ at $\theta_{0}=0^{\circ}$ in two cases: gold with $\epsilon=-9.89+i 1.05$ (calculations via the extinction theorem), and a perfect conductor up to the first iterate (single plus double scattering). At the bottom of the figure, the surface profile is depicted in an arbitrary scale. The agreement between both calculations is reasonably good. Note that, even though shadowing $^{53,55}$ is not taken into account in the iterative method, only at small portions of the surface slight discrepancies arise (see the curves at points $x$ on the left of the higher peak).

The important fact in these curves is the observation of large field enhancements [up to the value of 36 in terms of $|J(x)|^{2}$ for normal incidence] for both polarizations, accounted for by both methods. The fact that these enhancements appear for both polarizations in metals as well as in perfect conductors reveals that the excitation of SP can by no means be the cause of these intense fields. Actually, the reason must be the double-scattering contribution [recall that the single-scattering term-KAonly yields a value $|J(x)|^{2}=4$ everywhere on the surface]. From Fig. 9 it is evident that the larger values of the fields lie on points $x$ placed at both sides of the two deep valleys of the surface profile record, which is exactly 
where one expects double scattering to contribute more strongly. The occurrence of multiple scattering within the cavities of the rough surface gives rise to enhancements of both propagating and evanescent waves in the selvedge. In this connection, it should be remarked that intense line shapes in the nonradiative region have been obtained for deep gratings both for $s$ and $p$ polarization in this regime $T>\lambda .{ }^{10,56}$

In Sec. III A the phenomenon of enhanced backscattering was found by averaging the scattered intensity over 400 samples of metallic surfaces such as those of Fig. 9 (cf. Fig. 1). Therefore enhanced backscattering and the aforementioned surface-field enhancements, produced both by multiple scattering within the deep grooves of the surface, are intimately related.

\section{CONCLUSIONS}

In this paper we have studied multiple-scattering effects from one-dimensional randomly rough metallic surfaces. It has been seen that, for statistical parameters
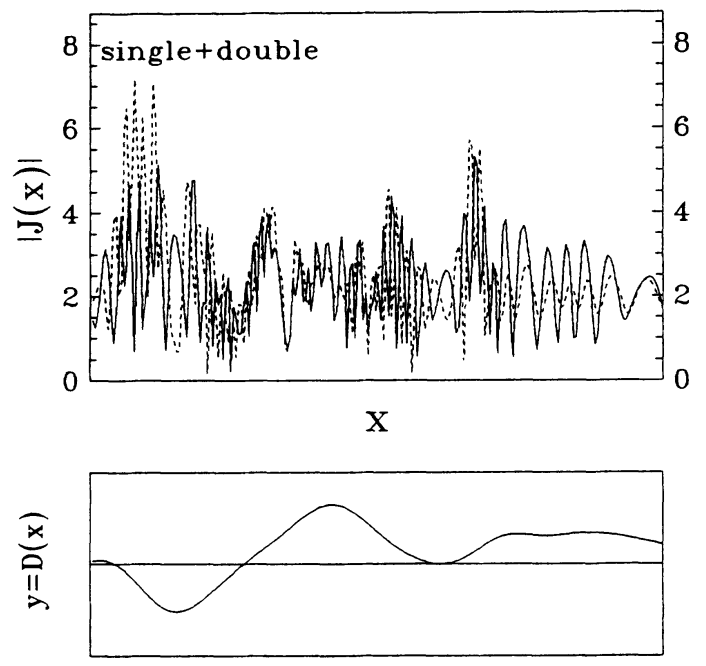

$\mathrm{X}$

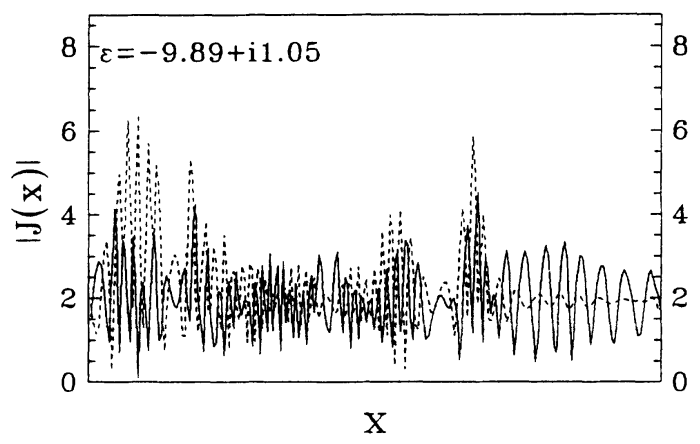

FIG. 9. Modulus of $J(x)$ for a sample length $L=2 a=27.81 \lambda$ extracted from a sequence with $T=3.16 \lambda$ and $\sigma=1.9 \lambda ; \theta_{0}=0^{\circ}$. Two cases are plotted: a perfect conductor iteratively solved up to double scattering, and the numerical result from the ET for $\epsilon=-9.89+i 1.05$. The sample profile in an arbitrary scale is included in between. Dashed line: $s$ polarization. Solid line: $p$ polarization.
$T$ and $\sigma$ larger than the wavelength, enhanced backscattering takes place, this being linked to double, and higher-order, scattering for both polarizations. Therefore, the state of polarization of the incident wave does not play such an important role in this regime of values of $T$. Moreover, the double-scattering contribution has revealed it is capable of producing surface-field enhancements especially noticeable in the cavities; this is also associated with the excitation of some kind of surface electromagnetic waves, although in the regime of $T$ and $\sigma$ the contribution of homogeneous (propagating) waves to these enhancements is indeed large.

By contrast, when $T$ and $\sigma$ are smaller than the wavelength, there are important effects due to the excitation of SP for $p$ polarization. We have shown that the observation, previously made, of a tiny backscatter peak in the mean scattered intensity is also linked to the existence of reflectance drops and of surface-field enhancements. However, these phenomena, although appreciable in statistical surfaces, are not as remarkable as in the case of metallic gratings, and neither is the angle of incident as critical in this respect. Also, we have made a theoretical interpretation (even if approximated) to account for the influence of the surface sample edges on the scattering from a flat interface in order to distinguish the effects due to the presence of corrugation from those, much weaker, produced by diffraction from the surface boundaries. A comparison of this analysis with the exact numerical results has been made, too.

\section{ACKNOWLEDGMENTS}

This work was supported by the Comision Interministerial de Ciencia y Tecnología under Grant No. PB-278. J. A. Sánchez-Gil acknowledges a grant from Ministerio de Educación y Ciencia. Discussions with J. C. Dainty and A. A. Maradudin, made possible by a NATO travel grant (No. 890528), and also with D. Maystre, are appreciated.

\section{APPENDIX}

In this appendix we address the calculation of the field above a flat interface $z=0$ from its angular spectrum representation, Eq. (10), for an incident $p$-polarized, finitely extended (i.e., of truncated wave front) plane wave. The angular spectrum is expressed by Eq. (11), where the corresponding structure factor [Eq. (12)] and Fresnel coefficient [Eq. (13b)] must be accounted for.

First, we rewrite $\mathscr{R}_{p}(K)$ (see, e.g., Ref. 10),

$$
R_{p}(K)=C(K)\left(\frac{1}{K-K_{\mathrm{SP}}}-\frac{1}{K+K_{\mathrm{SP}}}\right),
$$

where

$$
C(K)=\frac{1}{2 K_{\mathrm{SP}}} \frac{\left(\epsilon q-q^{\prime}\right)^{2}}{1-\epsilon^{2}} .
$$

On introducing Eqs. (11) and (12) into Eq. (10) one is led to 
$U^{(r)}(\mathbf{r})=\frac{1}{\pi} \int_{-\infty}^{\infty} d K \mathcal{R}_{p}(K) \frac{\sin \left[\left(K-K_{0}\right) a\right]}{K-K_{0}} e^{i(K x+q z)}$.

We divide the integral of Eq. (A3) into two integrals by expressing the sine function in terms of exponentials as follows:

$$
I=I_{1}+I_{2},
$$

where

$I_{1}=\frac{1}{2 \pi i} \int_{-\infty}^{\infty} d K \mathscr{R}_{p}(K) \frac{e^{-i K_{0} a}}{K-K_{0}} e^{i K(x+a)} e^{i q z}$,

$I_{2}=\frac{-1}{2 \pi i} \int_{-\infty}^{\infty} d K \mathcal{R}_{p}(K) \frac{e^{i K_{0} a}}{K-K_{0}} e^{i K(x-a)} e^{i q z}$.

Both integrals $I_{1}$ and $I_{2}$ may be evaluated by analytic continuation of the integrand and using an appropriate contour in the complex $K$ plane. When this is done, there are three poles to be taken into account: one on the real axis, $K_{0}=k_{0} \sin \theta_{0}$, and two others slightly shifted upwards $\left(K_{\mathrm{SP}}\right)$ and downwards $\left(-K_{\mathrm{SP}}\right)$, respectively, from the real axis. Cauchy's theorem can then be applied in a contour of integration given by the real axis and a semicircle of infinite radius, either in the lower or in the upper half-plane, depending on the location of the point $x$. We next discuss the result.

\section{1. $x<-a$}

We choose for both $I_{1}$ and $I_{2}$ the contour of integration in the lower half of the complex $K$ plane, so that the integrands have the appropriate behavior for $|K| \rightarrow \infty$ through the factors $e^{i k(x+a)}$ and $e^{i k(x-a)}$, respectively. Then, only the poles at $K_{0}$ and $-K_{\mathrm{SP}}$ contribute to the integrals. Moreover, the residues from $K=K_{0}$ in $I_{1}$ and in $I_{2}$ cancel each other; thus one is left uniquely with the contribution of the residue at $K=-K_{\mathrm{SP}}$. As a result, we arrive at

$$
\begin{aligned}
I_{1} & =S_{--}(x, z) \\
& =-\frac{C\left(-K_{\mathrm{SP}}\right)}{K_{\mathrm{SP}}+K_{0}} e^{-i K_{0} a} e^{-i K_{\mathrm{SP}}(x+a)} e^{-q_{\mathrm{SP}} z}, \\
I_{2} & =S_{+-}(x, z)=\frac{C\left(-K_{\mathrm{SP}}\right)}{K_{\mathrm{SP}}+K_{0}} e^{i K_{0} a} e^{-i K_{\mathrm{SP}}(x-a)} e^{-q_{\mathrm{SP}} z},
\end{aligned}
$$

$q_{\mathrm{SP}}$ being

$$
q_{\mathrm{SP}}=\left(K_{\mathrm{SP}}^{2}-k_{0}^{2}\right)^{1 / 2} .
$$

Equations (A6) and (A7) represent SP propagating to the left and starting at the boundaries $x=-a\left[S_{--}(x, z)\right]$ and $x=a\left[S_{+-}(x, z)\right]$ of the illuminated segment of length $L=2 a$ of the flat interface.

$$
\text { 2. }-a<x<a
$$

In this case, care has to be taken in the choice of the half-plane over which the contour of integration is used. The upper half-plane gives the correct behavior for the integrand of $I_{1}$ when $|K| \rightarrow \infty$ due to the factor $e^{i k(x+a)}$. Then the residues from $K=K_{0}$ and $K=K_{\mathrm{SP}}$ are calculated, thus yielding for $I_{1}$

$$
I_{1}=\frac{1}{2} \mathcal{R}_{p}\left(K_{0}\right) e^{i\left(K_{0} x+q_{0} z\right)}+S_{-+}(x, z),
$$

where

$S_{-+}(x, z)=\frac{C\left(K_{\mathrm{SP}}\right)}{K_{\mathrm{SP}}-K_{0}} e^{-i K_{0} a} e^{i K_{\mathrm{SP}}(x+a)} e^{-q_{\mathrm{SP}} z}$

is a SP that propagates to the right from $x=-a$. On the other hand, the first term in Eq. (A9) represents half the contribution from the specularly reflected field (recall that the incident field has the form $\left.e^{i\left(K_{0}-q_{0} z\right)}\right)$.

In order to obtain $I_{2}$, we integrate with the contour in the lower half-plane and, on introducing the residues from $K=K_{0}$ and $K=-K_{\mathrm{SP}}$, we arrive at

$$
I_{2}=\frac{1}{2} \mathcal{R}_{p}\left(K_{0}\right) e^{i\left(K_{0} x+q_{0} z\right)}+S_{+-}(x, z) .
$$

$S_{+-}(x, z)$ is the surface mode previously found in Eq. (A7).

$$
\text { 3. } x>a
$$

In this case, by integrating $I_{1}$ and $I_{2}$ over the contour in the upper half-plane, the contributions from the residues at $K=K_{\text {SP }}$ give

$$
\begin{aligned}
I_{1} & =S_{-+}(x, z), \\
I_{2} & =S_{++}(x, z) \\
& =-\frac{C\left(K_{\mathrm{SP}}\right)}{K_{\mathrm{SP}}-K_{0}} e^{+i K_{0} a} e^{i K_{\mathrm{SP}}(x-a)} e^{-q_{\mathrm{SP}} z} .
\end{aligned}
$$

$S_{-+}(x, z)$ was defined in Eq. (A10) and $S_{++}(x, z)$ is a SP propagating to the right from $x=a$. The contribution from $K=K_{0}$ vanishes, similarly to what occurs in the zone $x<-a$.
${ }^{1}$ Lord Rayleigh, Philos. Mag. 14, 70 (1907); Theory of Sound, 2nd ed. (Dover, New York, 1945), Vol. II, p. 89.

${ }^{2}$ U. Fano, J. Opt. Soc. Am. 31, 213 (1941).

${ }^{3}$ E. R. Méndez and K. A. O'Donnell, Opt. Commun. 61, 91 (1987); K. A. O’Donnell and E. R. Méndez, J. Opt. Soc. Am. A 4, 1194 (1987).

${ }^{4}$ A. J. Sant, J. C. Dainty, and M. J. Kim, Opt. Lett. 14, 1183
(1989).

${ }^{5}$ M. J. Kim, J. C. Dainty, A. T. Friberg, and A. J. Sant, J. Opt. Soc. Am. A 7, 569 (1990).

${ }^{6}$ N. García and E. Stoll, J. Opt. Soc. Am. A 2, 2240 (1985).

${ }^{7}$ M. Nieto-Vesperinas and J. M. Soto-Crespo, Opt. Lett. 12, 979 (1987).

${ }^{8}$ J. M. Soto-Crespo and M. Nieto-Vesperinas, J. Opt. Soc. Am. 
A 6, 367 (1989).

${ }^{9}$ A. A. Maradudin, E. R. Mèndez, and T. Michel, Opt. Lett. 14, 151 (1989).

${ }^{10}$ A. A. Maradudin, T. Michel, A. R. McGurn, and E. R. Méndez, Ann. Phys. (N.Y.) 203, 255 (1990).

${ }^{11}$ A. R. McGurn and A. A. Maradudin, Opt. Commun. 72, 279 (1989).

${ }^{12}$ M. Nieto-Vesperinas and J. M. Soto-Crespo, Phys. Rev. B 38, 7250 (1988).

${ }^{13}$ M. Nieto-Vesperinas and J. M. Soto-Crespo, Phys. Rev. B 39, 8193 (1988).

${ }^{14} \mathrm{~J}$. M. Soto-Crespo and M. Nieto-Vesperinas, Opt. Commun. 69, 185 (1989).

${ }^{15}$ M. Nieto-Vesperinas, J. A. Sánchez-Gil, A. J. Sant, and J. C. Dainty, Opt. Lett. 15, 1261 (1990).

16J. A. Sánchez-Gil and M. Nieto-Vesperinas, J. Opt. Soc. Am. A 8, 1270 (1991).

${ }^{17}$ M. Nieto-Vesperinas and J. A. Sánchez-Gil, J. Opt. Soc. Am. (to be published).

${ }^{18}$ Scattering in Volumes and Surfaces, edited by M. NietoVesperinas and J. C. Dainty (North-Holland, Amsterdam, 1990).

${ }^{19}$ A. K. Fung and M. F. Chen, J. Opt. Soc. Am. A 2, 2274 (1985).

${ }^{20}$ Y. Kuga and A. Ishimaru, J. Opt. Soc. Am. A 1, 831 (1984); Y. Kuga, L. Tsang, and A. Ishimaru ibid. 2, 616 (1985).

${ }^{21}$ L. Tsang and A. Ishimaru, J. Opt. Soc. Am. A 1, 836 (1984).

${ }^{22}$ L. Tsang and A. Ishimaru, J. Opt. Soc. Am. A 2, 1331 (1985).

${ }^{23}$ M. P. Van Albada and A. Lagendijk, Phys. Rev. Lett. 55, 2692 (1985).

${ }^{24}$ P. E. Wolf and G. Maret, Phys. Rev. Lett. 55, 2696 (1985).

${ }^{25}$ S. Etemad, R. Thompson, and M. J. Andrejco, Phys. Rev. Lett. 57, 575 (1986).

${ }^{26}$ E. Akkermans, P. E. Wolf, R. Maynard, and G. Maret, J. Phys. (Paris) 49, 77 (1988).

${ }^{27}$ P. Beckmann and A. Spizzichino, The Scattering of Electromagnetic Waves from Rough Surfaces (Macmillan, New York, 1963).

${ }^{28}$ P. Beckmann, in Scattering of Light by Rough Surfaces, edited by E. Wolf, in Progress in Optics VI (North-Holland, Amsterdam, 1961), pp. 55-69.

${ }^{29}$ F. G. Bass and I. M. Fuks, Wave Scattering from Statistically Rough Surfaces (Pergamon, Oxford 1979).

${ }^{30}$ S. O. Rice, Commun. Pure Appl. Math. 4, 4808 (1951).

${ }^{31}$ G. R. Valenzuela, IEEE Trans. Antennas Propag. AP-15, 552 (1967).

${ }^{32}$ D. Beaglehole and O. Hunderi, Phys. Rev. B 2, 309 (1970); 2, 321 (1970).

${ }^{33}$ V. Celli, A. Marvin, and F. Toigo, Phys. Rev. B 11, 1779 (1975); A. Marvin, F. Toigo, and V. Celli, ibid. 11, 2777 (1975).

${ }^{34}$ G. S. Agarwal, Phys. Rev. B 15, 2371 (1977).
${ }^{35}$ F. Toigo, A. Marvin, V. Celli, and N. R. Hill, Phys Rev. B 15, 5618 (1977).

${ }^{36}$ N. García, V. Celli, and M. Nieto-Vesperinas, Opt. Commun. 30, 279 (1979).

${ }^{37}$ M. Nieto-Vesperinas and N. Garcia, Opt. Acta 28, 1651 (1981).

${ }^{38}$ M. Nieto-Vesperinas, J. Opt. Soc. Am. 72, 539 (1982).

${ }^{39}$ H. Raether, Surface Polaritons on Smooth and Rough Surfaces and on Gratings, Springer Tracts on Modern Physics Vol. III (Springer-Verlag, Berlin, 1988).

${ }^{40}$ V. M. Agranovich and D. L. Mills, Surface Polaritons (North-Holland, Amsterdam, 1982).

${ }^{41}$ A. R. McGurn, A. A. Maradudin, and V. Celli, Phys. Rev. B 31, 4866 (1985).

${ }^{42}$ V. Celli, A. A. Maradudin, A. M. Marvin, and A. R. McGurn, J. Opt. Soc. Am. A 2, 2225 (1985).

${ }^{43}$ A. R. McGurn and A. A. Maradudin, J. Opt. Soc. Am. B 4, 910 (1987).

${ }^{44}$ M. C. Hutley and D. Maystre, Opt. Commun. 19, 431 (1976).

${ }^{45}$ D. Maystre and M. Neviere, J. Opt. (Paris) 8, 15 (1977).

${ }^{46}$ M. Neviere and R. Reinisch, Phys. Rev. B 26, 5403 (1982).

${ }^{47}$ N. E. Glass, A. A. Maradudin, and V. Celli, Phys. Rev. B 27, 5120 (1983); J. Opt. Soc. Am. 73, 1240 (1983).

${ }^{48}$ N. García, Opt. Commun. 45, 307 (1983).

${ }^{49}$ D. L. Mills and M. Weber, Phys Rev. B 26, 1075 (1982); M. Weber and D. L. Mills, ibid. 27, 2698 (1983).

${ }^{50}$ W. C. Meecham, J. Ration. Mech. Anal. 5, 323 (1956).

${ }^{51}$ E. G. Liszka and J. J. McCoy, J. Acoust. Soc. Am. 71, 1093 (1982).

${ }^{52}$ E. I. Thorsos, J. Acoust. Soc. Am. 83, 78 (1988).

${ }^{53}$ A. Ishimaru and J. S. Chen, J. Acoust. Soc. Am. 88, 1877 (1990).

${ }^{54}$ C. Makaskill, J. Opt. Soc. Am. A 8, 88 (1991).

${ }^{55}$ N. Bruce and J. C. Dainty, J. Mod. Opt. 38, 579 (1991).

${ }^{56}$ A. Wirgin and A. A. Maradudin, Phys. Rev. B 31, 5573 (1985).

${ }^{57}$ D. N. Pattanayak and E. Wolf, Opt. Commun. 6, 217 (1972).

${ }^{58}$ M. Saillard and D. Maystre, J. Opt. Soc. Am. A 7, 982 (1990); see also M. Saillard, Ph.D. thesis, University of Aix-Marseille III, 1990.

${ }^{59}$ D. W. Lynch and W. R. Hunter, in Handbook of Optical Constants of Solids, edited by E. D. Palik (Academic, New York, 1985), pp. 294 and 356.

${ }^{60} \mathrm{M}$. Born and E. Wolf, Principles of Optics, 6th ed. (Pergamon, Oxford, 1980), Sec. 1.5.

${ }^{61}$ J. Crowell and R. H. Ritchie, J. Opt. Soc. Am. 60, 794 (1970); J. M. Elson and R. H. Ritchie, Phys. Rev. B 4, 4129 (1971); Phys. Status Solidi. 62, 461 (1974).

${ }^{62}$ S. O. Sari, D. K. Cohen, and K. D. Scherkoske, Phys. Rev. B 21, 2162 (1980).

${ }^{63}$ A. A. Maradudin and D. L. Mills, Phys. Rev. B 11, 1392 (1975). 